\title{
CORACOCLAVICULAR LIGAMENT AUGMENTATION AT CLAVICLE DISTAL-THIRD FRACTURE TREATMENT
}

\author{
Bulent Karslioglu, Metin Uzun, Suleyman Semih Dedeoglu, Yunus Imren, \\ Ahmet Keskin
}

The aim: type $2 B$ clavicle fractures with conoid ligament rupture are considered unstable. Although surgical treatment is recommended as the standard treatment modality for type $2 \mathrm{~B}$ fractures, there is no consensus about the type of operative treatment. We aimed to evaluate results of surgical treatment with an anatomical distal clavicle plate using CC ligament augmentation.

Materials and methods: 15 patients that diagnosed with distal clavicle fractures, who underwent surgery for unstable type 2 fractures. The average patient age was 38 years (range 24-52 years). All patients were male; the right clavicle was injured in 10 patients whereas the left clavicle was injured in 5 cases. Surgical treatment was done with a distal clavicle anatomic locked plate augmentation (ZipTight ${ }^{\mathrm{TM}}$ ) at all cases. The mean follow-up period was 24 months (range, 12-40 months).

Results: bony union was achieved at a mean follow-up of 8 weeks (range 6-10 weeks). The mean Constant score was 97 (range, 92-100). There were no complications or no need to second operation. All patients achieved satisfactory full range of shoulder motion. Hardware removal was performed for prominence in one case after the union was completed. Conclusion: the augmented technique reported here, provides early motion, increased stability and anatomic healing compared to other conventional options. We recommend augmentative CC ligament repair techniques over the distal locking anatomic plate for type 2 fractures.

Keywords: distal clavicle, coracoclavicular, ligament, plate, fracture, type 2, surgery, anatomic healing, early motion

Karslioglu, B., Uzun, M., Dedeoglu, S. S., Imren, Y., Keskin, A. (2021). Coracoclavicular ligament augmentation at clavicle distal-third fracture treatment. ScienceRise: Medical Science, 3 (42), 00-00. doi: http://doi.org/10.15587/2519-4798.2021.229927

(C) The Author(s) 2021

This is an open access article under the Creative Commons CC BY license

\section{Introduction}

Distal clavicle fractures account for $15 \%$ of all clavicle fractures [1]. Neer classified distal clavicular fractures into three types according to fracture pattern and the relationship of the fracture line to the coracoclavicular (CC) ligaments and acromioclavicular (AC) joint. Type 1: coracoclavicular ligaments intact; type 2: coracoclavicular ligaments detached from the medial segment, but trapezoid intact to distal segment; type 3: intraarticular extension into the acromioclavicular joint [2].

Type 2 divided into 2 subtypes according to conoid ligaments injury. Type $2 \mathrm{~B}$ fractures with conoid rupture as a part of the CC ligaments are considered unstable. Unbalanced forces, such as arm weight and muscle tractions on the fracture site, are other factors in nonunion fractures of the distal third of the clavicle. Conservative treatment for type $2 \mathrm{~B}$ fractures results in $22-50$ $\%$ non-union rates, with half of the non-union cases being symptomatic $[3,4]$.

Although surgical treatment is recommended as the standard treatment modality for type $2 \mathrm{~B}$ fractures, no consensus has been reached on the type of the operative treatment, which includes using sutures, slings, Kirschner wires, CC screws, anatomic locking plates, tension band wiring, Knowles pins, anatomic AC plates, and radius distal locking plates [1, 3, 5-9].
Several problems, such as implant failure and necessity for hardware removal, are related to these methods. To our knowledge, no comparative clinical study of these treatment options has been conducted. One major issue to address is that the distal fragment is small, communicated and cannot accommodate enough (2-4) screws for fracture stability, and the role of the CC ligament in this type of fracture is usually underestimated whereas efforts are generally focused on fracture stability.

Recently, some authors have advocated new plates with CC ligament augmentation capabilities that were used to increase fracture stability [10]. Venjakop previously reported the treatment of an AC joint injury by a new CC ligament repair technique [11].

We aimed to evaluate results of surgical treatment with an anatomical distal clavicle plate using CC ligament augmentation.

\section{Materials and methods}

We prospectively reviewed 15 patients diagnosed with distal clavicle fractures who underwent surgery for unstable type 2 fractures according to X-ray examination and Neer classification from March 2009 to August 2012 (Fig. 1). All participants were informed and informed consent form was obtained. Study was approved by Acibadem University Institutional Review Board (14/10/2015-365). 
The average patient age was 38 years (range 24 52 years). All patients were male; the right clavicle was injured in 10 patients whereas the left clavicle was injured in 5 cases.

Only patients with acute surgical treatment (within 3 weeks of injury) with an anatomic distal clavicle locked plate augmentation (ZipTight ${ }^{\mathrm{TM}}$ ) and at least 1 year of follow-up were included in the study. The average time from injury to surgery was 3 days (range 1-7 days). The mean follow-up period was 24 months (range 12-40 months). Eight patients were injured in a cycling accident, five patients were injured in the ski, and two were injured falling off a horse.

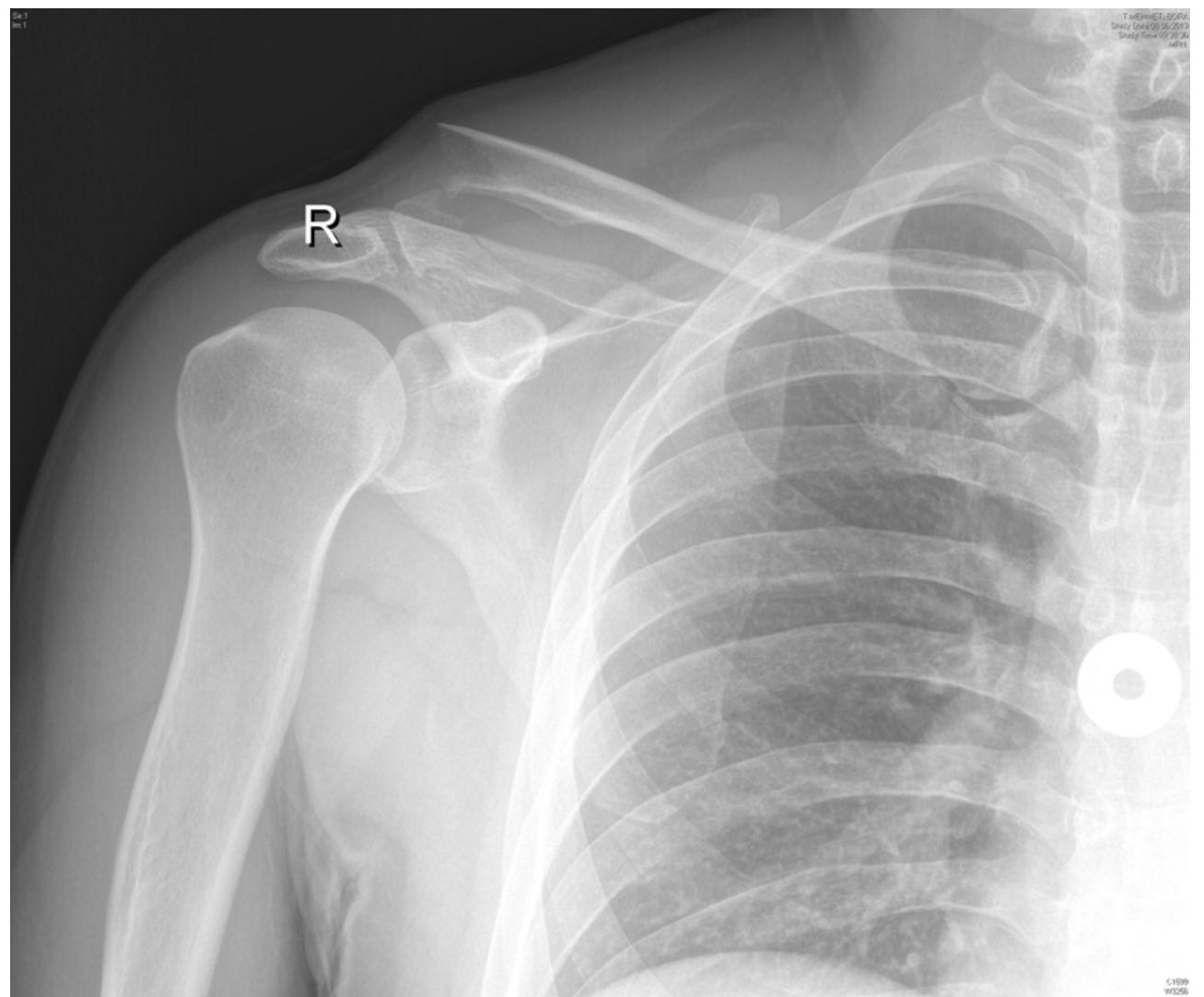

Fig. 1. 33 years man's shoulder AP x-ray image showing that clavicle distal communicated fracture

Surgical procedures were performed under general anesthesia with the patient in the beach-chair position. A standard anterosuperior approach to the clavicle was used. Care was taken to preserve the superior AC joint ligaments. After identification of the fracture site, all hematoma and debris were curetted and interposed soft tissue was removed. An anatomic distal clavicle plate was applied under fluoroscopy. Three or four screws were used to fix the distal part of the fracture and at least three screws were used at the medial part. The torn CC ligament was identified and reconstructed using a non-absorbable suture. The bullet was placed in the mid-aspect of the clavicle at $\sim 35 \mathrm{~mm}$ from the end of the clavicle over the plate. This location is midway for the attachments of the conoid and trapezoid coracoclavicular ligaments. Next, a 2.4-mm guide pin was drilled through the clavicle and coracoid. When the position of the guide pin was satisfied, a $4.5-\mathrm{mm}$ ToggleLoc ${ }^{\mathrm{TM}}$ reamer was used to drill over the pin through the clavicle and coracoid. After the bone tunnels were prepared, the ZipTight ${ }^{\mathrm{TM}}$ fixation device was passed through tunnels from the clavicle to coracoid. The ToggleLoc ${ }^{\mathrm{TM}}$ button exited the bone tunnel coracoid, and easily flipped onto the inferior side of the cortex. Zip strands were pulled while maintaining tension on the round top hat button down against the plate. After the round top hat button was seated, a ZipLoop puller was used for final tensioning of the device and was the rest of the incision was sutured (Fig. 2).

Postoperatively, a sling was used for 10 days. Passive range of shoulder motion began a couple of days after pain was relieved. Elevation of the arm above the shoulder was prohibited for three weeks. Functional outcome of the shoulder was evaluated using a Constant scoring system [12]. Union was evaluated radiologically. 


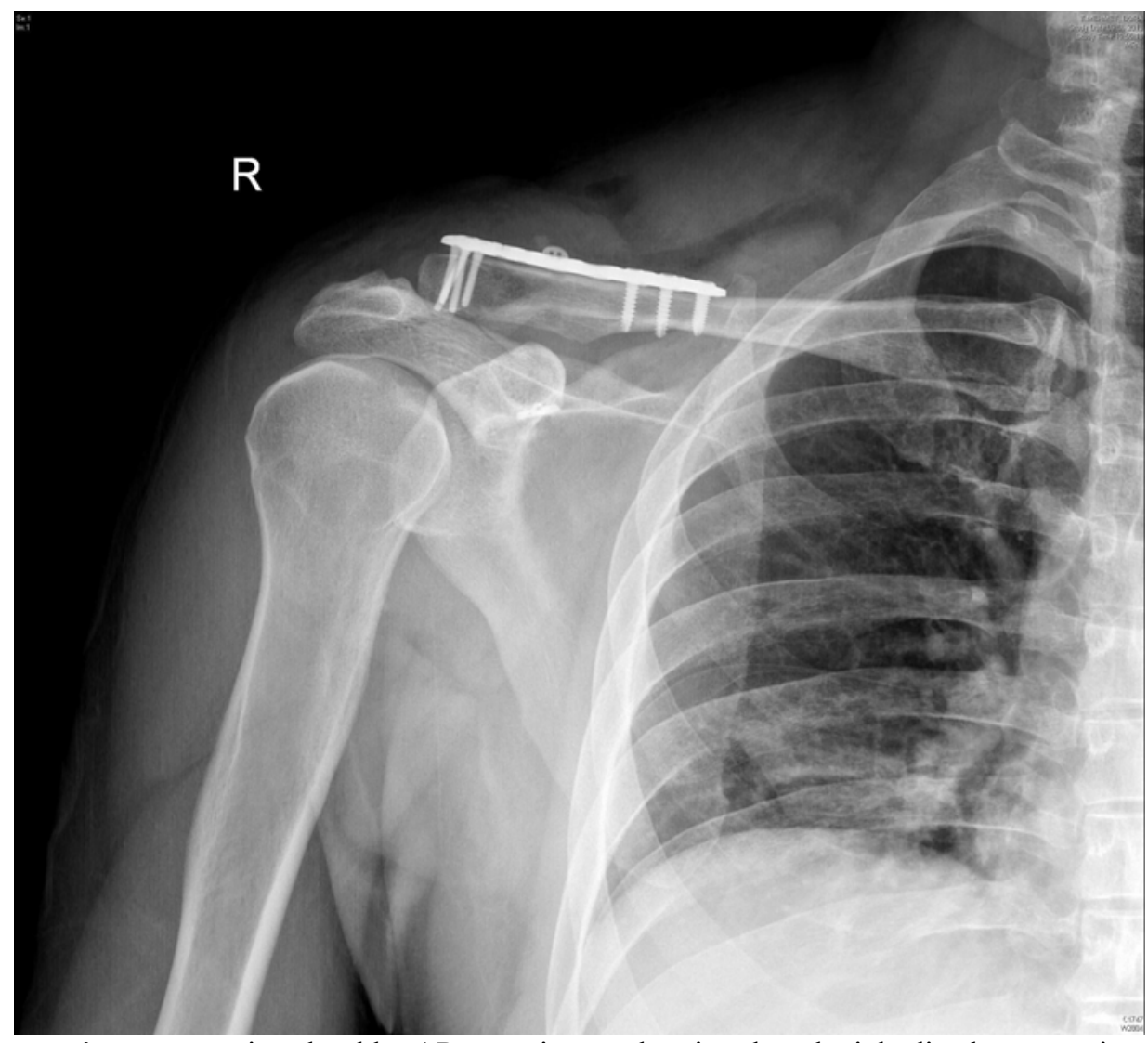

Fig. 2. 33 years man's postoperative shoulder AP x-ray image showing that clavicle distal communicated fracture that fixed anatomic plate augmented ZipTight

\section{Results}

Bony union was achieved at a mean follow-up of 8 weeks (range 6-10 weeks) (Fig. 3). The mean constant score was 97 (range 92-100). There were no complications, such as deep infection, non-union or malunion. All patients achieved satisfactory full range of shoulder mo- tion. The AC joint was observed, and remained reduced and normal without any radiological degeneration findings in all cases. Implant loosening was not seen in the plate or ZipTight ${ }^{\mathrm{TM}}$ device. Hardware removal was performed for prominence in one case after the union was completed.

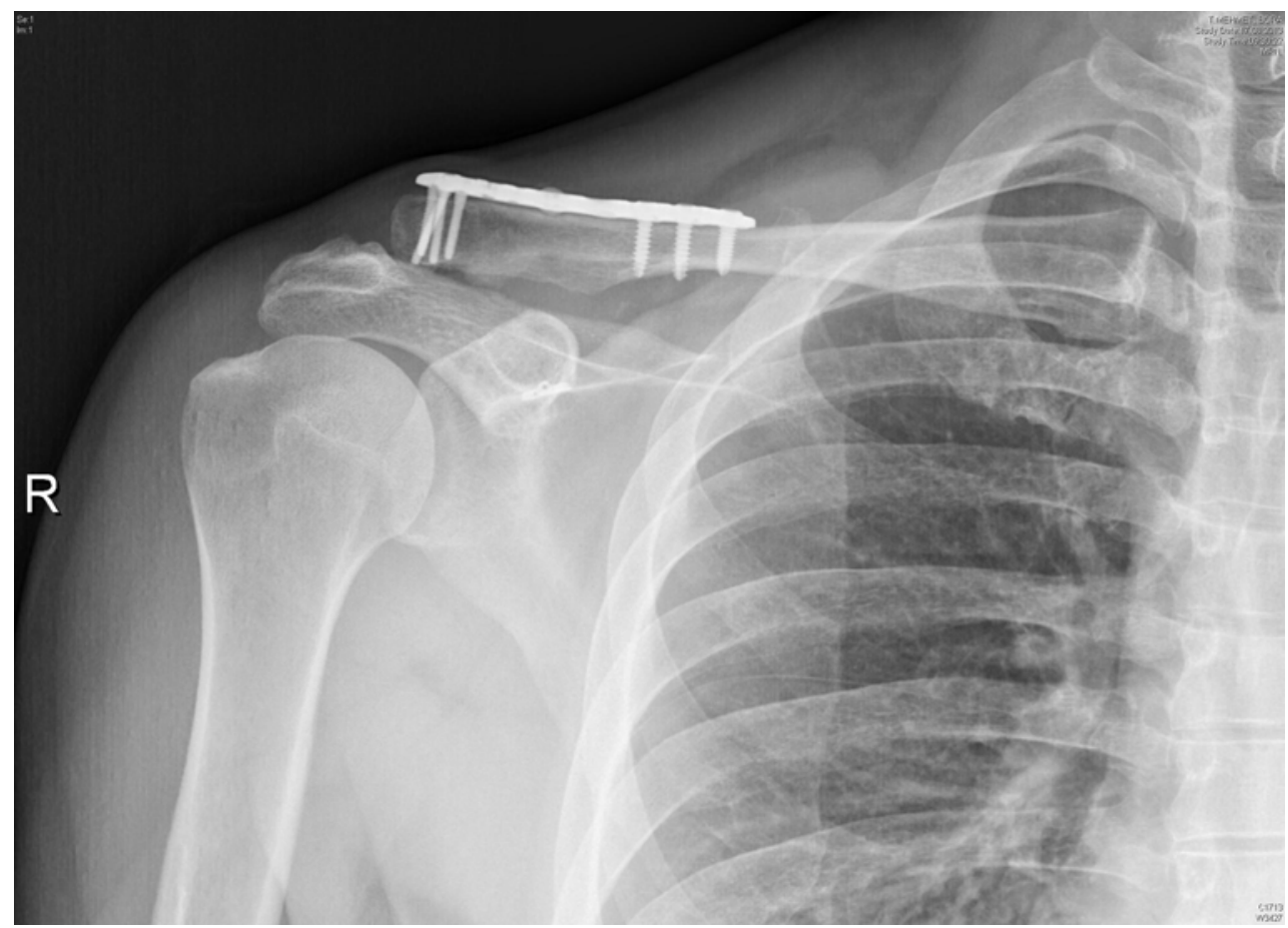

Fig. 3. 33 year's man's follow-up shoulder AP x-ray image showing that achieved union clavicle distal communicated fracture 


\section{Discussion}

Although surgical treatment is standard for type 2 fractures, ideal surgical treatment continues to be controversial [13]. The main issue regarding surgical treatment of type 2 fractures is union and early motion difficulties. Herein, we evaluated union, complications and results in a case series of patients treated with an anatomic plate augmented with Zip Tight ${ }^{\mathrm{TM}}$.

The distal clavicle fractures in both anatomy and position upon evaluation; the distal fragment is located in the posterior-inferior position due to attachment of the deltoid and trapezius; the proximal fragment is both superior and anterior due to the sternocleidomastoid and pectoralis major muscles. Although this muscle is able to function, it is difficult to open between the fractured fragments and results in a gap, causing the union issue. However, the main problem is ruptured CC ligaments, which many authors neglect during surgery [3, 13, 14]. Based on the literature, there are many surgical options for treatment of displaced distal clavicle fractures due to the union problem, including Kirschner wires, CC screws, anatomic locking plates, tension band wiring, Knowles pins, anatomic AC plates, radius distal locking plates, and augmentation screw or sutures [3, 13-17]. Despite the numerous techniques available, none method has been proven as superior [13].

Upon examination of the history of surgical treatments, Kirchner wires, Knowles pins and tension band wiring were the most useful options initially [3, 14, 18]. Klein and Shin reported AC joint arthrosis treated with Kirchner wires or Knowles pins [8, 15]. In addition, $\mathrm{Wu}$ recommends Kirchner wires and Knowles pins when the distal fragment is too small, but warned of the risk of migration of Kirchner wire [3]. However, with technological developments, locking plate technology has been proven as beneficial in fracture treatment with poor bone quality, as well as for short segments [10, 19]. Since the distal fragment is generally too small or comminuted for acute fractures, a new anatomic locking plate was introduced $[15,16]$. Multiple, divergent and locking screws in the distal fragment have increased pullout strength with use of an anatomic distal clavicle plate. This is especially important if the fragment is small or osteopenic. Andersen reported that use of multiple, divergent locking screws in the distal fragment provided sufficiently stable fixation [5].

Treatment of type 2 fractures is often difficult because the distal fragment is generally very small, communicated and cannot accommodate enough (2 to 4) screws for fracture stability. Sajid reported two cases requiring revision surgery due to plate pull-out and AC joint dislocation in patients treated using a distal anatomic clavicle plate [16]. A locking plate placed superiorly over the lateral end of the clavicle did not adequately neutralize all forces acting on the fracture site, which act predominantly to pull the fragment inferiorly.

Many authors have used clavicula-coracal cannulated screws, Knowles pins and coracoid suture augmentation to increase fracture stability; however, with increasing stability, it is sometimes disadvantageous because of the screw fixation requires additional surgery to remove the hardware caused by osteolysis [1]. Wang reported $12 \%$ asymptomatic $\mathrm{CC}$ heterotopic ossification with supported CC suture augmentation [14]. CC liga- ment repair or reconstruction with cerclage / Tightrope / button device / Zip Tight / Dacron graft for increased stability has been reported. Though these implants aim to achieve AC joint dislocation without fracture, Andersen reported similar results of a variety of non-plate fixation techniques for distal fractures [5]. All of these methods increase union rate and demonstrate similar results, but have their own specific complications. Shin reported that cutting the clavicle with TightRope leads to erosion of the clavicle with sutures [8]. Nevasier showed successful results with cerclage, but inadequate fixation for communicated fractures [20]. Rieser demonstrated that the combination of a locking plate with TightRope was superior to either method alone following biomechanical evaluation [10]. In this light, two important issues should be addressed:

1) acute type $2 b$ fractures are generally communicated and the distal fragment is too small, and ligament.

2) this type of fracture also injures the $\mathrm{CC}$

Therefore, the CC ligament should be treated simultaneously with fracture treatment. When an anatomical locking plate is chosen for surgery, Zip Tight ${ }^{\mathrm{TM}}$ may also be applied over the plate to prevent clavicle complications. This method proved successful (97 according to the constant score system), and active motion started 3-4 weeks after the surgery. Madsen and Schliemann also reported successful results of $\mathrm{CC}$ augmentation for distal clavicle fractures $[19,21]$. Salazar et. al also reported successful results in their comparative study which they compared locking plate fixation with and without CC augmentation [22]. Hazra et al. reported their results that $\mathrm{CC}$ augmentation added to angle stable plate osteosynthesis significantly reduced revision rates in lateral clavicula fractures [23]. In our study, we found the conclusion that $\mathrm{CC}$ augmentation leads to positive results in patients in accordance with the results of the literature.

Study limitations and prospects for future research. The low number of patients may be one of the limitations of our study. Conducting this study with a larger number of patients will increase the reliability of our study results. Evaluating the patients not only according to their bone union status, but also according to their return time to work will increase the reliability of this technique. Another limitation of our study is that the patients were not evaluated according to their return-to-work time.

\section{Conclusions}

The augmented technique reported herein provides increased stability and anatomic healing compared to conventional methods. In addition to increased stability, this new augmented technique provides early motion and allowance of daily activities immediately post-operation, with no need for a second procedure. We recommend augmentative $\mathrm{CC}$ ligament repair techniques over the distal locking anatomic plate for type 2 fractures.

\section{Conflict of interests}

The authors of this paper declare there is no conflict of interests.

\section{Funding}

None 


\section{References}

1. Ballmer, F., Gerber, C. (1991). Coracoclavicular screw fixation for unstable fractures of the distal clavicle. A report of five cases. The Journal of Bone and Joint Surgery. British Volume, 73-B (2), 291-294. doi: http://doi.org/10.1302/0301-620x.73b2.2005158

2. Neer, C. S. (1968). 5 Fractures of the Distal Third of the Clavicle. Clinical Orthopaedics and Related Research, 58, 43-50. doi: http://doi.org/10.1097/00003086-196805000-00007

3. Wu, C.-C. (2012). Tension Band Wiring versus Knowles Pinning for Non-Union of Type-2 Distal Clavicle Fractures. Journal of Orthopaedic Surgery, 20 (3), 297-301. doi: http://doi.org/10.1177/230949901202000306

4. Riiser, M. O., Molund, M. (2021). Long-term Functional Outcomes and Complications in Operative Versus Nonoperative Treatment for Displaced Midshaft Clavicle Fractures in Adolescents: A Retrospective Comparative Study. Journal of Pediatric Orthopaedics, 41 (5), 279-283. doi: http://doi.org/10.1097/bpo.0000000000001768

5. Andersen, J. R., Willis, M. P., Nelson, R., Mighell, M. A. (2011). Precontoured Superior Locked Plating of Distal Clavicle Fractures: A New Strategy. Clinical Orthopaedics \& Related Research, 469 (12), 3344-3350. doi: http://doi.org/10.1007/s11999-011-2009-5

6. Hohmann, E., Hansen, T., Tetsworth, K. (2012). Treatment of Neer type II fractures of the lateral clavicle using distal radius locking plates combined with TightRope augmentation of the coraco-clavicular ligaments. Archives of Orthopaedic and Trauma Surgery, 132 (10), 1415-1421. doi: http://doi.org/10.1007/s00402-012-1570-z

7. Liu, Q., Miao, J., Lin, B., Lian, K. (2012). Surgical Treatment for Unstable Distal Clavicle Fracture with Micromovable and Anatomical Acromioclavicular Plate. International Journal of Medical Sciences, 9 (4), 301-305. doi: http://doi.org/10.7150/ijms.4425

8. Shin, S.-J., Roh, K. J., Kim, J. O., Sohn, H.-S. (2009). Treatment of unstable distal clavicle fractures using two suture anchors and suture tension bands. Injury, 40 (12), 1308-1312. doi: http://doi.org/10.1016/j.injury.2009.03.013

9. Soliman, O., Koptan, W., Zarad, A. (2013). Under-coracoid-around-clavicle (UCAC) loop in type II distal clavicle fractures. The Bone \& Joint Journal, 95-B (7), 983-987. doi: http://doi.org/10.1302/0301-620x.95b7.31316

10. Rieser, G. R., Edwards, K., Gould, G. C., Markert, R. J., Goswami, T., Rubino, L. J. (2013). Distal-third clavicle fracture fixation: a biomechanical evaluation of fixation. Journal of Shoulder and Elbow Surgery, 22 (6), 848-855. doi: http://doi.org/10.1016/j.jse.2012.08.022

11. Venjakob, A. J., Salzmann, G. M., Gabel, F., Buchmann, S., Walz, L., Spang, J. T. et. al. (2013). Arthroscopically Assisted 2-Bundle Anatomic Reduction of Acute Acromioclavicular Joint Separations. The American Journal of Sports Medicine, 41 (3), 615-621. doi: http://doi.org/10.1177/0363546512473438

12. Constant, C. R., G. Murley, A. H. (1987). A Clinical Method of Functional Assessment of the Shoulder. Clinical Orthopaedics and Related Research, 214, 160-164. doi: http://doi.org/10.1097/00003086-198701000-00023

13. Stegeman, S. A., Nacak, H., Huvenaars, K. H., Stijnen, T., Krijnen, P., Schipper, I. B. (2013). Surgical treatment of Neer type-II fractures of the distal clavicle. Acta Orthopaedica, 84 (2), 184-190. doi: http://doi.org/10.3109/17453674.2013.786637

14. Wang, S.-J., Wong, C.-S. (2008). Extra-Articular Knowles Pin Fixation for Unstable Distal Clavicle Fractures. Journal of Trauma: Injury, Infection \& Critical Care, 64 (6), 1522-1527. doi: http://doi.org/10.1097/ta.0b013e3180593646

15. Klein, S. M., Badman, B. L., Keating, C. J., Devinney, D. S., Frankle, M. A., Mighell, M. A. (2010). Results of surgical treatment for unstable distal clavicular fractures. Journal of Shoulder and Elbow Surgery, 19 (7), 1049-1055. doi: http://doi.org/10.1016/j.jse.2009.11.056

16. Sajid, S., Fawdington, R., Sinha, M. (2012). Locking plates for displaced fractures of the lateral end of clavicle: Potential pitfalls. International Journal of Shoulder Surgery, 6 (4), 126. doi: http://doi.org/10.4103/0973-6042.106226

17. Bhatia, D., Page, R. (2012). Surgical treatment of lateral clavicle fractures associated with complete coracoclavicular ligament disruption: Clinico-radiological outcomes of acromioclavicular joint sparing and spanning implants. International Journal of Shoulder Surgery, 6 (4), 116. doi: http://doi.org/10.4103/0973-6042.106224

18. Zhang, F., Fu, Q., Li, Y., Lu, N., Chen, A., Zhao, L. (2021). Locking plate combined with titanium cable for Neer type II distal clavicle fractures. BMC Musculoskeletal Disorders, 22 (1). doi: http://doi.org/10.1186/s12891-021-04137-4

19. Schliemann, B., Roßlenbroich, S. B., Schneider, K. N., Petersen, W., Raschke, M. J., Weimann, A. (2013). Surgical treatment of vertically unstable lateral clavicle fractures (Neer $2 \mathrm{~b}$ ) with locked plate fixation and coracoclavicular ligament reconstruction. Archives of Orthopaedic and Trauma Surgery, 133 (7), 935-939. doi: http://doi.org/10.1007/s00402-013-1737-2

20. Neviaser, R. J. (1987). Injuries to the Clavicle and Acromioclavicular Joint. Orthopedic Clinics of North America, 18 (3), 433-438. doi: http://doi.org/10.1016/s0030-5898(20)30373-4

21. Madsen, W., Yaseen, Z., LaFrance, R., Chen, T., Awad, H., Maloney, M., Voloshin, I. (2013). Addition of a Suture Anchor for Coracoclavicular Fixation to a Superior Locking Plate Improves Stability of Type IIB Distal Clavicle Fractures. Arthroscopy: The Journal of Arthroscopic \& Related Surgery, 29 (6), 998-1004. doi: http://doi.org/10.1016/j.arthro.2013.02.024

22. Salazar, B. P., Chen, M. J., Bishop, J. A., Gardner, M. J. (2020). Outcomes after locking plate fixation of distal clavicle fractures with and without coracoclavicular ligament augmentation. European Journal of Orthopaedic Surgery \& Traumatology, 31 (3), 473-479. doi: http://doi.org/10.1007/s00590-020-02797-x

23. Dey Hazra, R.-O., Blach, R. M., Ellwein, A., Lill, H., Warnhoff, M., Jensen, G. (2021). Additional coracoclavicular augmentation reduces revision rates in the treatment of lateral clavicle fractures as compared to angle-stable plate osteosynthesis alone. Archives of Orthopaedic and Trauma Surgery. doi: http://doi.org/10.1007/s00402-021-03893-1

Received date 10.03.2021

Accepted date 20.04.2021

Published date 31.05.2021

Bulent Karslioglu, MD, Orthopedic Surgeon, Department of Orthopedics and Traumatology, HSU Prof. Cemil Tascioglu City Hospital, Darulaceze str., 25, Okmeydani Sisli, Istanbul, Turkey, 34382

E-mail: bukars@gmail.com

Metin Uzun, Associate Professor, Orthopedic Surgeon, Acibadem University Maslak Hospital, Darussafaka Buyukdere ave., 40, Sariyer, Istanbul, Turkey, 34457

E-mail: drmetinuzun@gmail.com

Suleyman Semih Dedeoglu, Associate Professor, Orthopedic Surgeon, Department of Orthopedics and Traumatology, HSU Prof. Cemil Tascioglu City Hospital, Darulaceze str., 25, Okmeydani Sisli, Istanbul, Turkey, 34382

E-mail: ssemihdedeoglu@gmail.com

Yunus Imren, Associate Professor, Orthopedic Surgeon, Department of Orthopedics and Traumatology, HSU Prof. Cemil Tascioglu City Hospital, Darulaceze str., 25, Okmeydani Sisli, Istanbul, Turkey, 34382

E-mail: yunusimren@gmail.com

Ahmet Keskin, MD, Orthopedic Surgeon, Department of Orthopedics and Traumatology, HSU Prof. Cemil Tascioglu City Hospital, Darulaceze str., 25, Okmeydani Sisli, Istanbul, Turkey, 34382 